\title{
Recurrencia de sismos en el Valle Central de Costa Rica
}

\section{Recurrence of Earthquakes in the Central Valley of Costa Rica}

\author{
Ronnie Quintero-Quintero ${ }^{l}$ \\ Hernán Porras-Espinoza ${ }^{2}$ \\ Universidad Nacional, Costa Rica
}

\begin{abstract}
Resumen
En los periodos 1910-1912, 1951-1955 y 1990-1993 se localizaron en el Valle Central de Costa Rica sismos superficiales importantes, por su magnitud y daños causados en la población, estructura y paisaje. La recurrencia de estos sismos es de aproximadamente cada 40 años y sucedieron entre 7 y 10 años, después de haber ocurrido los sismos mayores de subducción de 1904, 1941 y 1983 en la Península de Osa. En la zona central del país, también han ocurrido sismos esporádicos con magnitud de moderada a fuerte y que han causado daños. Por ejemplo, el sismo de Orotina de 1924, Pérez Zeledón de 1983, de Cinchona 2009 y Capellades del 2016. Si consideramos todos los sismos más importantes que han ocurrido en el interior de la parte central del país desde inicios del siglo XX y que han causado daños en el Valle Central; el período de recurrencia es de 21 años y la probabilidad de que el próximo se presente antes del 01/01/2038; 21 años después de haber ocurrido el sismo de Capellades el 01/12/2016, es mayor a 0.86 con un intervalo de confiabilidad del $95 \%$ de $[0.71,0.98]$.
\end{abstract}

Palabras clave: Valle Central, sismos, recurrencia, probabilidad condicional

\section{Abstract}

During the periods 1910-1912, 1951-1955, and 1990-1993, due to the magnitude and the damage caused to the population, structure, and landscape, important surface earthquakes were located in the Central Valley of Costa Rica. The recurrence of these earthquakes is approximately 40 years and

1 Académico, Observatorio Vulcanológico y Sismológico de Costa Rica, Universidad Nacional, Heredia, Costa Rica. Correo electrónico: rquinter@una.cr

2 Académico, Observatorio Vulcanológico y Sismológico de Costa Rica, Universidad Nacional. Heredia, Costa Rica. Correo electrónico: porrashernan@gmail.com

Este artículo corresponde a la ponencia presentada en el I Congreso Centroamericano de Ciencias de la Tierra y el Mar, realizado en el Hotel Aurola Holiday Inn en San José, Costa Rica, del 13 al 16 de noviembre de 2017. 
happened between seven and ten years, after the major subduction earthquakes of 1904, 1941, and 1983 in the Osa Peninsula. In the central region of the country, sporadic earthquakes have also occurred, with moderate to strong magnitude, and have caused damages, such as the Orotina earthquake in 1924, Peréz Zeledón in 1983, Cinchona in 2009 and Capellades in 2016. If we consider all the most important earthquakes that have occurred in the interior of the central part of the country since the beginning of the 20th century and that have caused damage in the Central Valley, the recurrence period is 21 years and the probability that the next one will occur before January 1, 2038, 21 years after the Capellades earthquake on December 1, 2016, is greater than 0.86 with a $95 \%$ confidence interval $[0.71,0.98]$.

Keywords: Central Valley, earthquakes, recurrence, conditional probability

\section{Introducción}

El estudio sísmico que por 34 años ha realizado la red del OVSICORI-UNA (Observatorio Vulcanológico y Sismológico de Costa Rica Universidad Nacional) en el país, nos indica que además de los sismos mayores y fuertes del tipo subducción a lo largo de la costa Pacífica de Costa Rica; tenemos sismicidad en la parte central del país que se presenta con sismos esporádicos moderados o fuertes; como los sismos de Cinchona del 2009 y el de Capellades del 2016, y otros que se dan agrupados en el tiempo (ver Tabla I y Figura 1). La sismicidad al interior del país es controlada e inducida por los sismos de subducción del Pacífico costarricense, tal y como lo sugiere el modelo de sliver motion propuesto para el noroeste (NW) de Costa Rica (Montero et al., 2017).

Los hipocentros de los sismos registrados en la zona central del país muestran sismicidad en la placa continental desde el antearco hasta el trans-arco, con profundidades que llegan hasta la base de la corteza; la sismicidad muestra la existencia de una zona en el antearco, asociada al promontorio de Herradura, donde la sismicidad es poca o nula (ver Figura 1). Esta zona de sismicidad en la parte central del país se conoce como Cinturón Deformado del Centro de Costa Rica (Marshall, 2000). 
Figura 1. Mapa que muestra los epicentros de sismos en Costa Rica; registrados por la red del OVSICORI-UNA desde el 2010 hasta finales del 2017 (Figura de la izquierda).
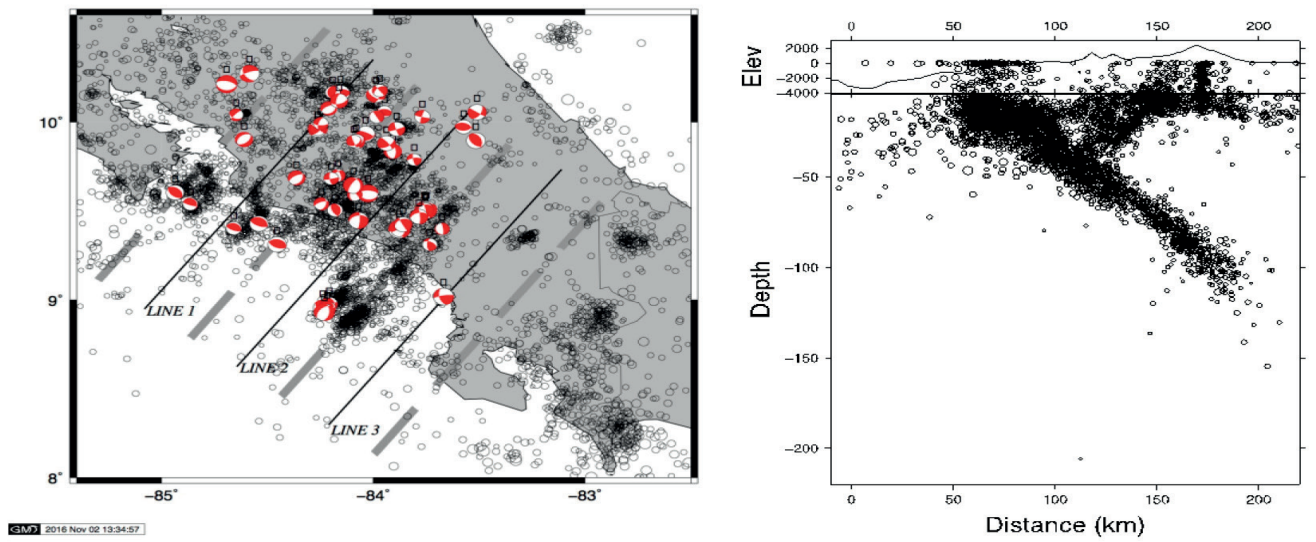

Fuente: OVSICORI-UNA, 2010-2017

Los mecanismos focales se muestran por medio de esferas de color rojo y la proyección de la falla en el hemisferio inferior de la esfera focal. A la derecha, se muestra la proyección de estos sismos en la Línea 1; el eje de coordenada del gráfico muestra dos medidas, una en metros y la otra en $\mathrm{km}$; desde la superficie hasta $4 \mathrm{~km}$ debajo del nivel del mar está dada en metros y de ahí hacia abajo en $\mathrm{km}$. Los sismos proyectados son los que se encuentran entre las dos líneas grises y extremas del mapa epicentral. Los sismos en ambas figuras se representan por círculos no rellenos de color negro y con tamaño acorde a su magnitud.

La recurrencia de los sismos moderados y fuertes que se localizan al interior de la placa continental y que han afectado el Valle Central del país, es el tema de estudio en este trabajo. Queremos dar respuesta a las interrogantes: ¿cuándo ocurren los sismos destructivos en el Valle Central? como los de Cartago de 1910, y ¿cuál es la probabilidad de que ocurra un nuevo sismo que cause daños en la zona central del país después de haber ocurrido el sismo de Capellades del 2016? ¿están relacionados los sismos destructivos en el Valle Central con los sismos mayores de subducción que ocurren bajo la Península de Nicoya o la de Osa? Para tratar de contestar 
esas preguntas, recurrimos a los catálogos sísmicos que existen a nivel local y global.

Para Costa Rica, se conoce que la velocidad de convergencia de la placa del Coco, respecto al Bloque de Panamá, es de 8-9 cm/a, con una dirección de convergencia de $20^{\circ}-30^{\circ}$ desde el norte (De Mets et al., 2010). La velocidad al interior del país es mucho menor, comparada con la velocidad de convergencia Coco - Bloque de Panamá y es de $\sim 2.07 \mathrm{~cm} / \mathrm{a}$ y con una dirección de $43.5^{\circ}$ en la estación de río Macho, Orosí, Cartago (RIMA); como se puede ver en la Figura 2, que muestra la velocidad en las componentes norte, este y vertical de esa estación. Para una falla como la que generó el terremoto de Cartago de 1910, cercana al sitio de RIMA; si consideramos un sismo característico de magnitud 6.4 y que la taza promedio de desplazamiento de la falla sea de $\sim .5 \mathrm{~cm} \mathrm{a}^{-1}$; el período de recurrencia es mayor a los 100 años. 
Figura 2. Serie de tiempo para la componente norte, este y vertical de la estación continua de GPS (CGPS), RIMA.
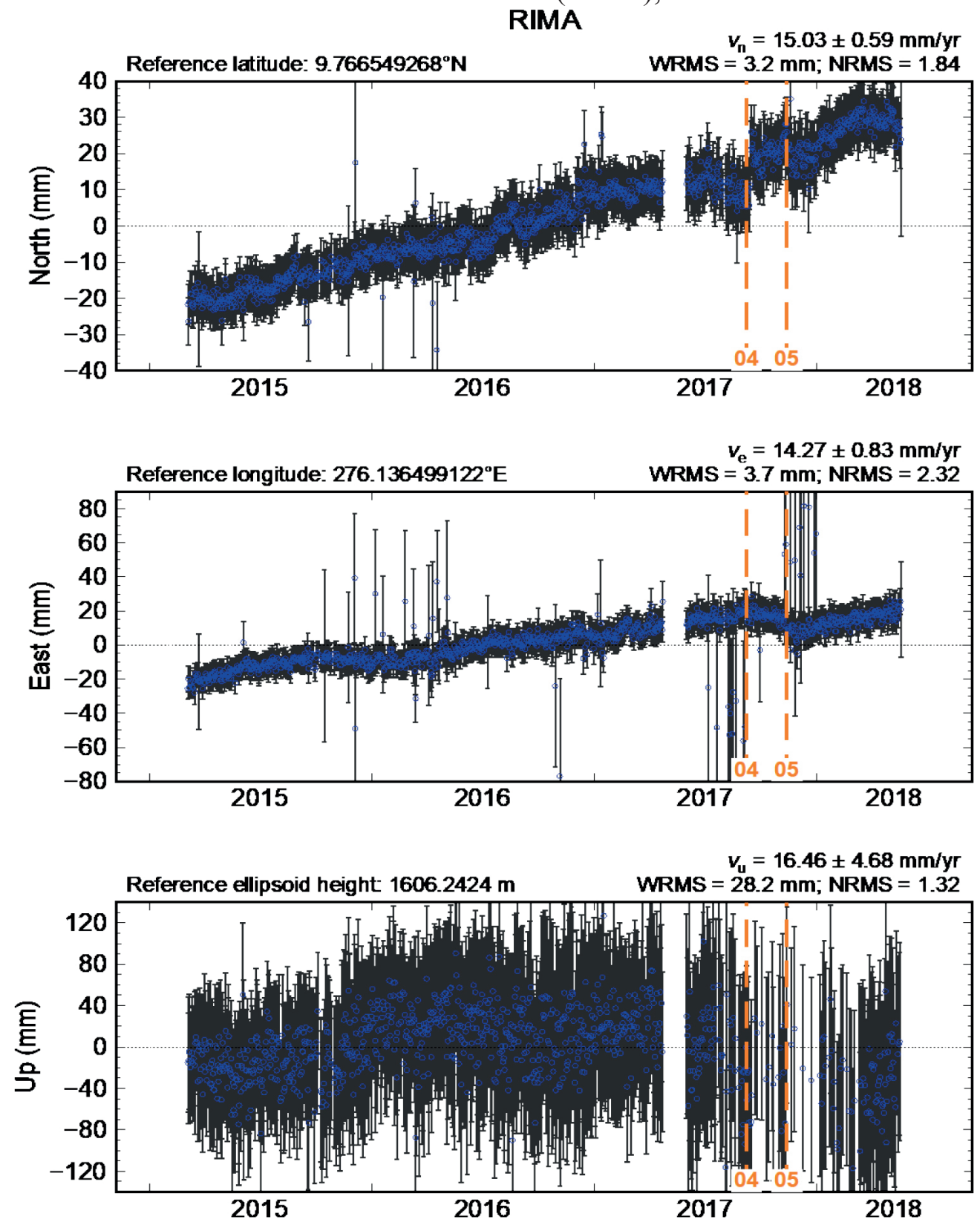
Se indica la velocidad norte Vn, velocidad este Ve y velocidad vertical $\mathrm{Vu}$ (milímetro/año). La figura muestra algunos huecos donde la estación CGPS no trabajó. La ubicación de la estación está indicada por latitud, longitud y altura en la parte de arriba e izquierda del gráfico.

\section{Período de recurrencia}

Haciendo uso de los catálogos sísmicos para el país (ver Tabla I); podemos decir que, desde que hay información más confiable de éstos a partir del siglo XX; varios sismos destructivos localizados en el Valle Central se han presentado en forma de ciclos sísmicos. Estos ciclos se han presentado de 1910 a 1912, con varios terremotos al interior, siendo el más dañino el de Cartago; luego está el segundo ciclo del siglo XX, que se da entre los años del 1951 a 1955, y el tercero entre los años 1990 a 1993 (Protti et al., 2001).

Tabla I. Sismicidad Histórica de Costa Rica. Las intensidades están dadas en la escala Mercalli Modificada (MM). AAAA/MM/DD se refiere a año, mes y día; HH:MI:SEC a la hora en que ocurrió el sismo (hora UTC) y MAG a la magnitud.

\begin{tabular}{|c|l|l|l|l|}
\hline $\begin{array}{c}\text { AAAA } \\
\text { /MM/DD }\end{array}$ & HH:MI:SE & MAG & Ubicación & \multicolumn{1}{|c|}{ Característica } \\
\hline $1900 / 06 / 21$ & $20: 52: 00$ & 7.1 & $\begin{array}{l}17.4 \mathrm{~km} \text { sur } \\
\text { de Nicoya, } \\
\text { Guanacaste }\end{array}$ & $\begin{array}{l}\text { Precedido por sismos menores. Causó } \\
\text { daños generalizados en Guanacaste. } \\
\text { Daños en la iglesia de Filadelfia y } \\
\text { en lochos de varias casas de ese } \\
\text { pueblo. Daños menores en Nicoya, } \\
\text { La Cruz y Puntarenas. Daños en la } \\
\text { iglesia de Rivas, Nicaragua }\end{array}$ \\
\hline $1904 / 12 / 20$ & $05: 44: 18$ & 7.5 & $\begin{array}{l}\text { En zona sur } \\
\text { de Costa } \\
\text { Rica }\end{array}$ & $\begin{array}{l}\text { El área donde se reporta el sismo } \\
\text { se extendió más allá de los límites } \\
\text { del país. Mo }=2.3 \text { x e27 dyne -cm. } \\
\text { Causó pequeños daños en San José, } \\
\text { Sentido VII en Bocas del Toro, David, } \\
\text { Bugaba, todos de Panamá; en Limón } \\
\text { y Coto, Costa Rica. Localización } \\
8.7^{\circ} \text { N, 83.6 }{ }^{\circ} \text { W }\end{array}$ \\
\hline
\end{tabular}




\begin{tabular}{|c|c|c|c|c|}
\hline $\begin{array}{c}\text { AAAA } \\
\text { /MM/DD }\end{array}$ & HH:MI:SE & MAG & Ubicación & Característica \\
\hline 1910/04/13 & $18: 37: 00.0$ & 5.8 & $\begin{array}{l}\text { Sur este de } \\
\text { San José. } \\
\text { (Tablazo) }\end{array}$ & $\begin{array}{l}\text { Sismo violento, sentido en el Valle } \\
\text { Central, principalmente en Cartago } \\
\text { y San José. Objetos caídos en } \\
\text { casas y tiendas. Daños producto } \\
\text { de deslizamientos. Seguido de } \\
\text { muchas réplicas. Intensidad de IX en } \\
\text { Coralillo, Cartago. }\end{array}$ \\
\hline $1910 / 05 / 05$ & 00:50:00.0 & 6.4 & Cartago & $\begin{array}{l}\text { Segunda destrucción de Cartago, } \\
\text { reportan } 600 \text { fallecidos; la primera } \\
\text { destrucción fue en 1841. Reportes } \\
\text { de daños también en San José, } \\
\text { Heredia, San Rafael, Tarrazú y } \\
\text { Orosí. Intensidad máxima de VII en } \\
\text { Cartago Centro. Se considera que el } \\
\text { sismo estuvo asociado a la falla de } \\
\text { Aguacaliente, ubicada en Cartago. }\end{array}$ \\
\hline $1911 / 08 / 29$ & 04:06:00.0 & 5.8 & $\begin{array}{l}\text { Bajos } \\
\text { del Toro, } \\
\text { Valverde } \\
\text { Vega, } \\
\text { Alajuela }\end{array}$ & $\begin{array}{l}\text { Daños en casas de Toro Amarillo, } \\
\text { daños menores en Grecia y Alajuela. } \\
\text { Sentido fuerte en San José, pero } \\
\text { sin reportes de daños. Numerosos } \\
\text { deslizamientos cercanos al Volcán } \\
\text { Poás. Región epicentral se estimó } \\
\text { a } 6-9 \text { km NO del cráter del Volcán } \\
\text { Poás. Intensidades de IX en Toro } \\
\text { Amarillo, VII en Naranjo y Grecia, } \\
\text { VI en Alajuela y V en San José. }\end{array}$ \\
\hline $1912 / 02 / 21$ & $08: 20: 00.0$ & 5.6 & Tres Ríos & $\begin{array}{l}\text { Daños en casas de Tres Ríos. Sentido } \\
\text { fuerte en el Valle Central. Intensidad } \\
\text { VI-VII Tres Ríos de la Unión, } \\
\text { Cartago. }\end{array}$ \\
\hline 1912/06/06 & $18: 40: 00.0$ & 5.1 & $\begin{array}{l}\text { Sarchí, Toro } \\
\text { Amarillo }\end{array}$ & $\begin{array}{l}\text { Daños en la zona de Sarchí, San } \\
\text { Pedro, Grecia y Toro Amarillo, } \\
\text { deslizamientos, avalanchas y } 7 \\
\text { muertos. Intensidad máxima de IX en } \\
\text { zona epicentral, VII en Toro Amarillo, } \\
\text { VI en Naranjo y Grecia. }\end{array}$ \\
\hline
\end{tabular}


Ronnie Quintero-Quintero - Hernán Porras-Espinoza Recurrence of Earthquakes in the Central Valley of Costa Rica

\begin{tabular}{|c|c|c|c|c|}
\hline $\begin{array}{c}\text { AAAA } \\
/ \text { MM/DD }\end{array}$ & HH:MI:SE & MAG & Ubicación & Característica \\
\hline $1924 / 03 / 04$ & 10:07:42.0 & 7.0 & Orotina & $\begin{array}{l}\text { Daños en toda la región occidental } \\
\text { del Valle Central, más de } 70 \\
\text { fallecidos. Intensidad máxima de X } \\
\text { en zona epicentral; IX en Orotina y } \\
\text { San Mateo; VIII en Puriscal, Atenas } \\
\text { y Esparza. }\end{array}$ \\
\hline $1941 / 12 / 05$ & $20: 46: 57.6$ & 7.5 & $\begin{array}{l}\text { Península de } \\
\text { Osa }\end{array}$ & $\begin{array}{l}\text { Sentido fuerte en la zona fronteriza } \\
\text { con Panamá y en el Valle Central de } \\
\text { Costa Rica. Intensidad máxima de IX } \\
\text { en Puerto Cortés, Puerto Jiménez y } \\
\text { Península de Osa. Intensidad VIII en } \\
\text { Golfito y Palmar. }\end{array}$ \\
\hline 1948/11/19 & $01: 04: 24.0$ & 6.3 & $\begin{array}{l}\text { Región } \\
\text { Central } \\
\end{array}$ & Daños leves en el Valle Central \\
\hline $1950 / 10 / 05$ & $16: 09: 30.5$ & 7.7 & $\begin{array}{l}\text { Península de } \\
\text { Nicoya }\end{array}$ & $\begin{array}{l}\text { Daños en Puntarenas y Valle Central. } \\
\text { La Catedral y algunas casas en Nicoya } \\
\text { con daños; además, se reportaron } \\
\text { daños en Filadelfia, Santa Cruz y } \\
\text { Paraíso de Santa Cruz. Intensidades } \\
\text { máximas de VIII en Nicoya. No cobró } \\
\text { vidas humanas. }\end{array}$ \\
\hline $1951 / 08 / 22$ & $05: 41: 33.4$ & 5.7 & $\begin{array}{l}\text { Sur de } \\
\text { Cartago }\end{array}$ & $\begin{array}{l}\text { Destrucción en Paraíso y Orosí; daños } \\
\text { en San José. Intensidad máxima de } \\
\text { VII en Paraíso, Cartago. }\end{array}$ \\
\hline $1952 / 12 / 30$ & $12: 07: 03.0$ & 6.2 & $\begin{array}{l}\text { Nor-oeste } \\
\text { del Volcán } \\
\text { Irazú. } \\
\text { Terremoto } \\
\text { de Patillos }\end{array}$ & $\begin{array}{l}\text { Deslizamientos en las faldas del } \\
\text { Volcán Irazú, } 21 \text { muertos. Coronado } \\
\text { es el pueblo con mayor afectación. } \\
\text { Daños menores en Cartago. } \\
\text { Intensidad máxima de VIII en San } \\
\text { Isidro de Coronado. }\end{array}$ \\
\hline $1955 / 09 / 01$ & $17: 33: 00.0$ & 5.3 & $\begin{array}{l}\text { Toro } \\
\text { Amarillo - } \\
\text { Zarcero }\end{array}$ & $\begin{array}{l}\text { Daños en Toro Amarillo y norte de } \\
\text { Alajuela, } 10 \text { muertos. Muchos de los } \\
\text { daños producto de deslizamientos. } \\
\text { Daños se reportaron en Cartago, } \\
\text { Naranjo y otras partes al norte de } \\
\text { Alajuela. Intensidad máxima VII en } \\
\text { zona epicentral. }\end{array}$ \\
\hline
\end{tabular}




\begin{tabular}{|c|c|c|c|c|}
\hline $\begin{array}{c}\text { AAAA } \\
\text { /MM/DD }\end{array}$ & HH:MI:SE & MAG & Ubicación & Característica \\
\hline $1983 / 04 / 03$ & 02:50:03 & 7.4 & Golfito & $\begin{array}{l}\text { Daños en Golfito, Zona Sur y } \\
\text { Valle Central, } 1 \text { muerto. Sismo de } \\
\text { Subducción. Intensidad máxima de } \\
\text { VIII en Sierpe, San Francisco de } \\
\text { Tinoco y Villa Colón y de VII en } \\
\text { Golfito, Ciudad Neilly, Río Claro y } \\
\text { Palmar. }\end{array}$ \\
\hline $1983 / 07 / 03$ & $17: 14: 26.8$ & 6.3 & $\begin{array}{l}\text { Pérez } \\
\text { Zeledón }\end{array}$ & $\begin{array}{l}\text { Daños al Norte de San Isidro del } \\
\text { General, deslizamientos, } 1 \text { muerto. } \\
\text { Sismo Intraplaca. Intensidad Máxima } \\
\text { VIII-IX en zona epicentral. Daños en } \\
\text { la carretera Interamericana entre San } \\
\text { Isidro - Cartago. Por la ubicación } \\
\text { produjo muchos deslizamientos en la } \\
\text { zona epicentral. }\end{array}$ \\
\hline $1989 / 02 / 26$ & $12: 21: 11.0$ & 5.3 & Los Santos & $\begin{array}{l}\text { Daños y deslizamientos en Acosta, } \\
\text { San José. Sismo Intraplaca. Intensidad } \\
\text { Máxima de VI en zona epicentral y V } \\
\text { en San José y Cartago Centro. Ml } 4.4 \\
\text { y Mw 5.3. }\end{array}$ \\
\hline $1990 / 06 / 30$ & $14: 51: 07.2$ & 5.5 & Puriscal & $\begin{array}{l}\text { Enjambre sísmico en la zona } \\
\text { de Puriscal. Daños en Puriscal, } \\
\text { deslizamientos en Fila de Picagres. } \\
\text { Sismo Intraplaca. Intensidad máxima } \\
\text { de VI-VII en Santiago de Puriscal y V } \\
\text { en San José Centro. }\end{array}$ \\
\hline $1990 / 12 / 22$ & $11: 27: 54.1$ & 5.9 & Puriscal & $\begin{array}{l}\text { Daños en el Valle Central, } \\
\text { especialmente en el sector oeste } \\
\text { (Alajuela, La Guácima, Atenas, } \\
\text { Cuidad Colón, Turrúcares, Puriscal), } \\
1 \text { muerto. Intensidad máxima de VIII } \\
\text { en Santiago de Puriscal y VII en } \\
\text { Alajuela Centro. }\end{array}$ \\
\hline 1991/08/09 & $09: 33: 49.3$ & 5.2 & Los Santos & $\begin{array}{l}\text { Daños en Frailes de Desamparados, } \\
\text { San Pablo de León Cortés y Corralillo } \\
\text { de Cartago. Sismo por Fallamiento } \\
\text { Local de Corrimiento Lateral. } \\
\text { Intensidad máxima de VII en Frailes } \\
\text { y Corralillo de Desamparados, San } \\
\text { José. }\end{array}$ \\
\hline
\end{tabular}


Ronnie Quintero-Quintero - Hernán Porras-Espinoza Recurrence of Earthquakes in the Central Valley of Costa Rica

\begin{tabular}{|c|c|c|c|c|}
\hline $\begin{array}{c}\text { AAAA } \\
/ \mathbf{M M} / \mathbf{D D}\end{array}$ & HH:MI:SE & MAG & Ubicación & Característica \\
\hline $1992 / 03 / 07$ & $01: 53: 36.8$ & 6.5 & $\begin{array}{l}1.5 \mathrm{~km} \\
\text { sur este de } \\
\text { Naranjo }\end{array}$ & $\begin{array}{l}\text { Daños en la zona epicentral. Ocurre } \\
\text { dentro de la placa del Coco a } 71 \mathrm{~km} \text { de } \\
\text { profundidad. Sentido con intensidad } \\
\text { V en San Ramón, Naranjo, Atenas, } \\
\text { Alajuela y Heredia. }\end{array}$ \\
\hline $1993 / 07 / 10$ & $20: 41: 00.0$ & 5.8 & $\begin{array}{l}12 \mathrm{~km} \text { sur } \\
\text { de Turrialba }\end{array}$ & $\begin{array}{l}\text { Daños en la zona epicentral y } \\
\text { Turrialba. Sismo de Pejiballe, } \\
\text { intraplaca de corrimiento lateral. } \\
\text { Intensidad máxima VII en Pejibaye y } \\
\text { Humo de Jiménez, Cartago. }\end{array}$ \\
\hline 2009/01/08 & $19: 21: 34.0$ & 6.1 & $\begin{array}{l}\text { Cinchona, } \\
\text { Alajuela }\end{array}$ & $\begin{array}{l}6 \text { km este del Volcán Poás. Intensidad } \\
\text { máxima deVIII-IXen Cinchonayzona } \\
\text { epicentral. Causó deslizamientos, } \\
\text { casas destruidas, pueblo de Cinchona } \\
\text { destruido, corte de agua y luz; } \\
\text { edificios públicos dañados. Dañó } \\
\text { edificios en Alajuela centro y Heredia } \\
\text { Centro (en la Universidad Nacional } \\
\text { se tuvo que demoler edificio por } \\
\text { daños causados). } 30 \text { Personas entre } \\
\text { fallecidas y desaparecidas. Bosques } \\
\text { afectados, ríos con daños. Perdidas } \\
\text { en Turismo y en la producción. Sismo } \\
\text { de Corrimiento Lateral, intraplaca } \\
\text { Caribe, superficial. Aceleración } \\
\text { máxima en Heredia Centro de } 17 \% \\
\text { de g y en Alajuela Centro de } 22 \% \\
\text { de g; ambas a } 22 \text { km del epicentro. } \\
\text { En Batán de Limón con aceleración } \\
\text { máxima de } 13 \% \text { de g, } 90 \text { km del } \\
\text { epicentro. }\end{array}$ \\
\hline
\end{tabular}




\begin{tabular}{|c|c|c|c|c|}
\hline $\begin{array}{c}\text { AAAA } \\
/ \text { MM/DD }\end{array}$ & HH:MI:SE & MAG & Ubicación & Característica \\
\hline $2012 / 09 / 05$ & $14: 42: 03$ & 7.6 & $\begin{array}{l}\text { Océano } \\
\text { Pacífico, } \\
\text { frente a } \\
\text { Sámara, } \\
\text { Nicoya, } \\
\text { Guanacaste }\end{array}$ & $\begin{array}{l}\text { Daños sentido fuerte en la mayor } \\
\text { parte del territorio Nacional. Casas } \\
\text { con fracturas; igual que edificios } \\
\text { gubernamentales. Hospital de la } \\
\text { Anexión en Nicoya y Monseñor } \\
\text { Sanabria, Puntarenas con problemas } \\
\text { estructurales. Municipalidad de Santa } \\
\text { Cruz con daños. Afectó zonas alejadas } \\
\text { de Grecia, Zarcero y vecindades del } \\
\text { Volcán Arenal. Intensidad máxima de } \\
\text { VIII-IX. }\end{array}$ \\
\hline 2016/12/01 & $00: 25: 19.5$ & 5.2 & $\begin{array}{l}\text { Capellades } \\
\text { de Alvarado, } \\
\text { Cartago }\end{array}$ & $\begin{array}{l}\text { Daños en algunas casas cercanas } \\
\text { al epicentro, causó pequeños } \\
\text { deslizamientos que quedaron en } \\
\text { fondo de quebradas y riachuelos } \\
\text { y agrietamiento de suelo. Provocó } \\
\text { que Fuertes lluvias durante la última } \\
\text { semana de setiembre } 2017 \text { (antes de } \\
\text { la tormenta NATE del } 5 \text { de octubre) } \\
\text { causara desbordamientos de ríos, } \\
\text { caída de puentes y deslizamientos en la } \\
\text { zona de Cartago. El sismo no disparó } \\
\text { cambios en la actividad volcánica del } \\
\text { Volcán Turrialba. Muchas réplicas el } \\
\text { primer y segundo día y decayendo } \\
\text { rápidamente conforme pasaron los } \\
\text { días; ya al quinto día pocas réplicas. } \\
\text { Intensidades de IV-VII en zona } \\
\text { epicentral (MM). }\end{array}$ \\
\hline
\end{tabular}

Fuente: Guendel, F. (1986); Pacheco \& Sykes (1992), Ambraseys \& Adams (1996), Montero (1999); Protti et al. (2001); Fernández \& Montero (2002); Linkimer, at al. (2013); Segura et al. (2014); International Seismological Centre (ISC, 2015); Campos \& Quintero (2017); OVSICORIUNA (2018); RSN (2018).

Los tres ciclos sísmicos mencionados arriba, están correlacionados en el tiempo con los sismos mayores de subducción que ocurrieron en 1904, 1941 y 1983 en el sureste del país bajo la Península de Osa; pero, con un retardo de 7 a 10 años. La correlación temporal con los terremotos de Nicoya es menor, además, que los sismos de subducción del Pacífico norte tienen la 
frontera noreste (NE) de cuñas, en las fallas de Hacienda-Chiripa ubicadas en la Cordillera Volcánica de Guanacaste (Montero et al., 2017); por lo que la correlación con los sismos del Valle Central no es directa.

Para saber cómo están relacionados los sismos mayores de subducción de la Zona Sur con los sismos que producen daño al interior del país; debemos de procurar conocer el período de retorno de los sismos que han causado daños en el Valle Central. Revisando los datos mostrados en la Tabla I, han sido 20 los sismos que han causado daños en la zona central del país (ver Figura 3).

Figura 3.

Sismos que han causado daños en el Valle Central desde 1900

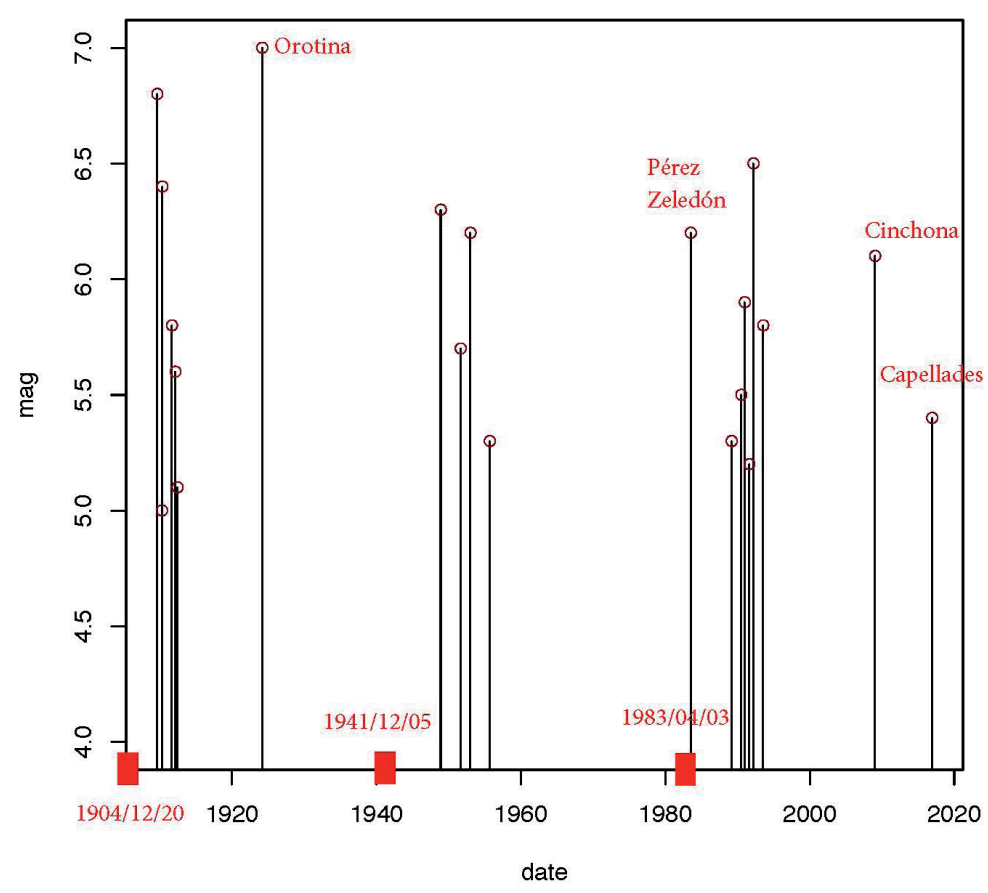

Gráfico que muestra la ocurrencia (fecha) de los sismos desde inicios del siglo XX en Costa Rica y que han afectado la zona central del país con su respectiva magnitud. También se indican con rectángulos de color rojo 
los sismos de OSA de 1904, 1941 y 1983. Para cuatro de los sismos; se indica en el gráfico el nombre como se nombró al sismo.

Tomando en cuenta los sismos mostrados en la Figura 3, y agrupando con una media de tiempo aquellos sismos que se encuentran cercanos en el tiempo; obtenemos seis fechas de sismos que han causado daños en el centro del país; a saber 01/01/1911, 04/03/1924, 26/04/1952, 22/12/1990, 08/01/2009, 01/12/2016. Estos sismos nos dan un período de retorno de 21 años; si nosotros no tomamos en cuenta los sismos de Cinchona y Capellades (Figura 3), por encontrarse alejados en el tiempo del sismo 03/04/1983, el período de retorno es de 27 años, y si, además, descartamos el sismo de Orotina, el período es de 40 años. Esta es la única forma que coincida el período de retorno de los sismos de la Zona Sur, con los sismos que han causado daños en el Valle Central del país. Lo que notamos de la Figura 3 , es que existen tres periodos 1910-1912, 1951-1955 y 1990-1993; donde hay concentración de sismos superficiales que han afectado la parte central del país y que se encuentran muy cercanos en el tiempo con los sismos mayores de la zona sur de 1904, 1941 y 1983.

Conociendo el período de retorno de los sismos que causaron daños en el Valle Central; ¿cuál es la probabilidad de que ocurra un sismo dentro de 21 años, partiendo del sismo de Capellades del 01/12/2016?

Para contestar esta pregunta, usando un período de retorno de 21 años; como encontramos arriba y conociendo que el último sismo que causó daños en la zona central fue el sismo de Capellades; obtenemos que para el 2037, existe una alta probabilidad (64\%) de que ocurra un sismo que cause daño en el Valle Central del país (ver Figura 4). Para calcular la probabilidad de ocurrencia de un sismo importante que pueda ocasionar daños en el Valle Central es $\mathrm{p}=1 / \mathrm{T}$, donde $\mathrm{T}$ es el período de retorno; entonces la probabilidad de que no ocurra cualquier año es (1-p) y al cabo de $n$ años es $(1-p)^{n}$ y de que ocurra es $\mathrm{P}=1-(1-\mathrm{p})^{\mathrm{n}}$. Esta última fórmula es la que se utiliza para realizar el gráfico mostrado en la figura 4. 
Figura 4.

$64 \%$ de Probabilidad de ocurrir en el 2037

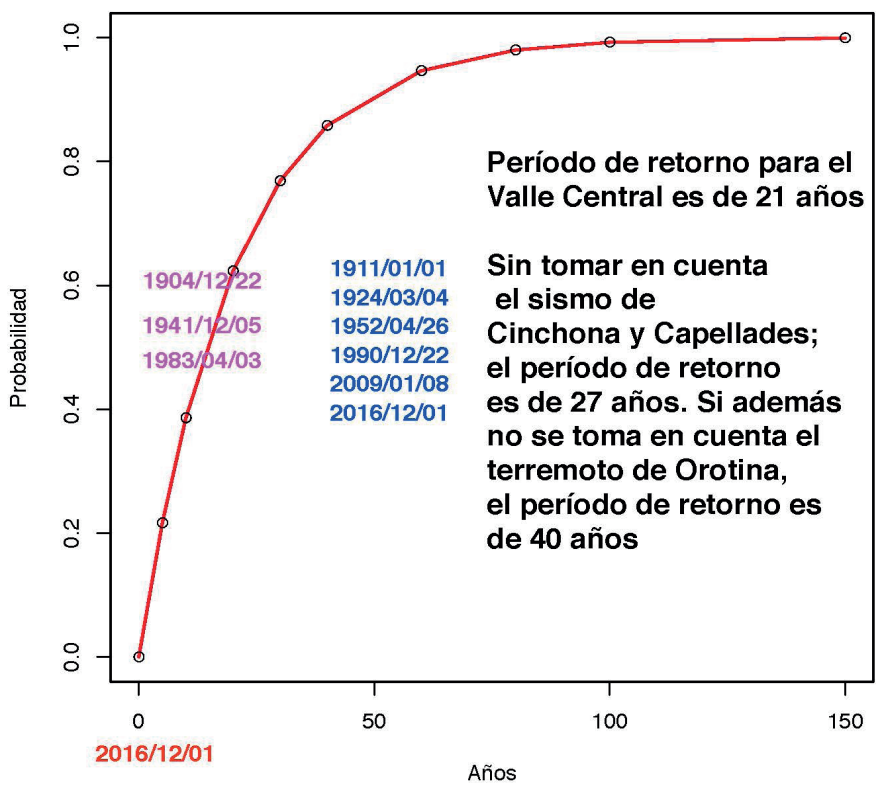

Gráfico que muestra la probabilidad de ocurrencia de un sismo que cause daños en el Valle Central de Costa Rica; tomando como referencia el último sismo el de Capellades del 01/12/2016 y un período de retorno de 21 años.

Ahora, ¿cuál sería la probabilidad de que ocurra un sismo en los siguientes 21 años; después de ocurrido el sismo de Capellades del 01/12/2016 y que cause daños en el Valle Central del país? Para estimar la probabilidad condicional de recurrencia de sismos destructivos en el Valle Central, usamos el modelo empírico de Savage (1994); en general, el modelo empírico de Savage (1994) es una distribución beta con parámetros $\mathrm{P}(\mathrm{p} \mid \mathrm{m}, \mathrm{n})=\operatorname{Beta}(\mathrm{m}+1, \mathrm{n}-\mathrm{m}+1)$. De la Tabla I (ver también Figura 3), seleccionamos aquellos sismos superficiales que han causado algún grado de daños en el Valle Central; los cuales son: 1909.02, 1910.28, 1910.34, 1911.66, 1912.14, 1912.43, 1924.17, 1948.88, 1951.64, 1952.99, 1955.66, 1983.50, 1989.15, 1990.49, 1990.97, 1991.60, 1992.18, 1993.52, 2009.02, 2016.91; con intervalos de recurrencia de $0.65,0.06,1.31,0.48,0.29,11.73,24.70$, 
$2.75,1.35,2.66,27.83,5.64,1.34,0.48,0.63,0.58,1.34,15.49$ y 7.88 años. La probabilidad de que el siguiente sismo ocurra antes del 2038.00: implicando un intervalo de recurrencia de 21.08 años, se calcula usando 17 intervalos de recurrencia que cumplen esta condición de un total de 19. Por lo que la media $\overline{\mathrm{p}}$ es de 0.86 , donde el intervalo de confianza del $95 \%$ está dado por $[0.71,0.98]$; por lo que podemos considerar que existe una alta probabilidad de que ocurra un sismo que cause daños en el Valle Central del país, antes del 01/01/2038. ¿Qué sucede, si consideramos que el sismo no ocurrió hasta el 23/05/2018? Desde el 01/12/2016 hasta el 23/05/2018 corresponde a un intervalo de 1.47 años. Eliminando los intervalos de recurrencia menores a 1.47; tenemos que 6 intervalos cumplen la condición de un intervalo de recurrencia de 21.08 años de un total de 8; por lo que la distribución es $\beta(7,3)=\mathrm{P}(\mathrm{p} \mid 6,8)$, dando como resultado una media $\overline{\mathrm{p}}_{\mathrm{de}}$ 0.7 con un intervalo de confianza del $95 \%$ de [0.45, 0.97]; mostrando una probabilidad e incertidumbre alta de que ocurra un sismo que cause daños en el Valle Central.

\section{Conclusiones}

Costa Rica ha presentado periodos de levantamiento y de subsidencia, como resultado de la interacción entre los procesos tectónicos regionales y los ciclos sísmicos de la zona de subducción. Estos ciclos sísmicos en la actualidad se han identificado al norte en la Península de Nicoya (en los años, 1900, 1950 y 2012) y en la Península de Osa (en los años, 1904, 1941 y 1983). La frecuencia y magnitud de estos sismos depende de diversos factores en la placa subductante, tales como asperezas, edad, velocidad y ángulo de convergencia y ángulo de subducción, entre otros. Los sismos más importantes por su magnitud, que han ocurrido en el interior del país, se dieron en los periodos de 1910-1912, 1951-1955 y 1990-1993. Estos sismos tienen una recurrencia de aproximadamente 40 años, similar al ciclo sísmico en la Península de Osa. Sin embargo, los sismos en el interior del país ocurren entre 7 y 10 años, después de haber ocurrido los eventos de la Península de Osa. Ahora, si consideramos los sismos superficiales que han ocurrido en Costa Rica desde inicios del siglo XX y que se han caracterizado por producir daños en el Valle Central del país; aunque con fuentes sísmicas diferentes; obtenemos 20 sismos de moderados a fuertes, 
desde 1900 hasta el 2016. Esos 20 sismos tienen un período de recurrencia de 21 años, que es menor a los 40 años de recurrencia de los sismos mayores de subducción en la Península de Osa. Tomando en consideración esos 20 sismos; podemos calcular que la probabilidad de que ocurra un sismo fuerte en el Valle Central en los próximos 21 años es bastante alta. Por lo tanto, aunque hay una estrecha relación en el tiempo entre los sismos mayores en la zona sur y ciclos de sismos en el Valle Central de aproximadamente 40 años, siempre han ocurrido otros sismos esporádicos que nos indican un período de recurrencia menor; que indica que no hay que esperar los sismos mayores de subducción en el SE de Costa Rica para que se produzcan sismos moderados o fuertes al interior del país y que causen daños en el Valle Central.

\section{Agradecimientos}

Las figuras se generaron usando el programa computacional GMT (Wessel and Smith, 1995), y R (R Core Team, 2013). Los datos fueron tomados del catálogo sísmico del ISC (2015), OVSICORI-UNA (2018) y RSN (2018).

\section{Referencias}

Ambraseys, N.N. \& Adams, R.D. (1996). Large-magnitude central American earthquakes, 1898-1994. Geophys. J. Int., 127 (1996), 665-692. Recuperado de https://doi.org/10.1111/j.1365-246X.1996.tb04046.x Campos, D. \& Quintero, R. (2017). Mapa de Intensidades e implicaciones socioambientales del sismo de Capellades. Nov. 30, 2016; Costa Rica. I Congreso Centroaméricano de Ciencias de la Tierra y el Mar. Llevado a cabo del 13 al 16 de noviembre, 2017. San José, Costa Rica.

De Mets, C., Gordon, R.G. \& Argus, D.F. (2010). Geologically Current Plate Motions. Geophys. J. Int. 181: 1-80.

Fernánez, M. \& Montero, W. (2002). Fallamiento y Sismicidad del área entre Cartago y San José, Valle Central de Costa Rica. Revista Geológica de América Central, 26: 25-37.

Guendel, F. (1986). Seismotectonic of Costa rica: an analytical view of the souther terminus of the Middle American trench, $\mathrm{PhD}$ thesis, UCSC, USA. 
International Seismological Centre. (2015). On-line Bulletin, Recuperado de http://www.isc.ac.uk, Internatl. Seismol. Cent., Thatcham, United Kingdom.

Marshall, J.S., Fisher, D.M. \& Gardner, T.W. (2000). Central Costa Rica deformed belt: kinematics of diffuse faulting across the western $\mathrm{Pa}-$ nama block, Tectonics, 19, 468-492.

Montero, W. (1999). El terremoto del 4 de marzo de 1924 (Ms 7.0): Un gran temblor interplaca relacionado al límite incipiente entre la placa Caribe y la microplaca de Panamá. En Revista Geológica de América Central, 22: 25-62.

Montero, W., Lewis, J. C. \& Araya, M. C. (2017). The Guanacaste Volcanic Arc Sliver of Northwester Costa Rica. Scientific Reports, SP 1797, V1 7, IS 1. DOI: 10.1038/s41598-017-01593-8.

Linkimer, L., Arroyo, I., Mora, M., Vargas, A., Soto, G. J., Barquero, R., Rojas, W., Taylor, W. \& Taylor, M. (2013). El terremoto de Sámara (Costa Rica) del 5 de Setiembre del 2012 (Mw 7,6). Revista Geológica de América Central, 49, 73-82.

Observatorio Vulcanológico y Sismológico de Costa Rica, Universidad Nacional (2018). Recuperado de http://www.ovsicori.una.ac.cr/index. $\mathrm{php} /$ sismologia/sismicidad-historica. Último acceso 22 mayo, 2018.

Pacheco, J. F. \& Sykes, L. R. (1992). Seismic moment catalogue of large, shallow earthquakes 1900 to1989. Bull. Seism. Soc. Am., 82 (3), 1306-1349.

Protti, M, Guendel, F. \& Malavassi, E. (2001). Evaluación del Potencial Sísmico de la Península de Nicoya. Editorial Fundación UNA, Heredia, C. R.

Red Sismológica Nacional (RSN) (2018). Sismos históricos. Recuperado de http://rsn.ucr.ac.cr/actividad-sismica/sismos-historicos. Último acceso 08 junio, 2018.

Savage, J. C. (1994). Empirical earthquake probabilities from observed recurrence intervals. Bulletin of the Seismological Society of America 84, 219-221.

Segura, J. D., Quintero, R., Burgoa, R. B. \& Jiménez, U. W. (2014). Análisis de la actividad sísmica en Costa Rica durante el 2010 y resumen de los eventos sísmicos más importantes presentados en Costa 
Rica de 1983-2012. Revista Geográfica de América Central, 52(2), 71-102.

R Core Team (2013). R: A language and environment for statistical computing. R Foundation for Statistical Computing, Vienna, Austria. Recuperado de URL http://www.R-project.org/.

Coast and Geodetic Survey, Washington, D.C, Science Service \& Asociación Jesuita Sismológica (1935). Seismological notes. Bulletin of the Seismological Society of America,25(4), 387-389. Recuperado de https://pubs.geoscienceworld.org/ssa/bssa/article/25/4/387/115140/ seismological-notes

Wessel, P. \& Smith, W.H.F. (1995). New version of the generic mapping tools released. EOS 76, 329. 